\begin{tabular}{|c|l|}
\hline Title & $\begin{array}{l}\text { New Method for Preparing Monodispersed Nanocrystall line Silicalite via Hydrothermal Synthesis in } \\
\text { Water/Surfactant/Oil Solution }\end{array}$ \\
\hline Author(s) & Tago, Teruoki; Nishi, Mieko; Kouno, Y ousuke; Masuda, Takao \\
\hline Citation & $\begin{array}{l}\text { Chemistry Letters, 33(8), 1040-1041 } \\
\text { https://doi.org/10.1246/1.2004.1040 }\end{array}$ \\
\hline Issue Date & 2004 \\
\hline Doc URL & http://hdl.handle.net/2115/70864 \\
\hline Type & article \\
\hline File Information & Chem.Lett.33.1040.pdf \\
\hline
\end{tabular}

Instructions for use 


\title{
New Method for Preparing Monodispersed Nanocrystalline Silicalite via Hydrothermal Synthesis in Water/Surfactant/Oil Solution
}

\author{
Teruoki Tago, Mieko Nishi, Yousuke Kouno, and Takao Masuda \\ Division of Materials Science \& Engineering, Graduate School of Engineering, Hokkaido University, \\ N13W8, Kita-Ku, Sapporo 060-8628
}

(Received April 14, 2004; CL-040414)

\begin{abstract}
Monodispersed silicalite nanocrystals with a diameter of $80-150 \mathrm{~nm}$ were successfully prepared in water-surfactant-oil solution. It was found that the ionicity of the surfactants used in preparation affected the crystallinity and structure of the silicalite, and that silicalite nanocrystals could be obtained when using a nonionic surfactant. Furthermore, silicalite nanocrystals with a diameter of approximately $80 \mathrm{~nm}$ could be obtained in even a high concentration region of $\mathrm{Si}$ source.
\end{abstract}

Zeolites, crystalline aluminosilicates, have been widely used in industry as heterogeneous catalysts and adsorbents. Moreover, zeolites crystals contain micropores with diameters almost equal to those of lighter hydrocarbons. The uniform size and shape of the micropores allows zeolites to be used as shape-selective catalysts. However, as compared with the size of micropores exhibiting a molecular-sieving effect, the crystal size of zeolites is very large, approximately $1-3 \mu \mathrm{m}$. When using a zeolite as a shape-selective catalyst, the diffusion rates of reactants, such as hydrocarbon molecules, within the zeolite crystals are relatively low in comparison with the reaction rates. This resistance to mass transfer causes the limitation of the reaction rates, low selectivity of intermediates and coke deposition, leading to the short lifetime of the catalysts. In order to overcome these problems, it will be necessary to achieve faster mass transfer of the reactants in the crystals; to this end, nanometer-sized zeolite crystals would be a promising solution.

The zeolite nanocrystals have attracted the considerable attention of many researchers. ${ }^{1-4}$ The syntheses of several types of zeolites with different nanometer sizes, such as silicalite-1, ZSM-5, A-type and Y-type, have been reported. Recently, micellar solutions or surfactant-containing solutions have been used for the preparation of zeolite nanocrystals. Hosokawa and Oki have reported a preparation method for the nanosized Atype zeolite using a nonionic surfactant and polyethylene glycol as crystallization inhibitors. ${ }^{5}$ We have also successfully prepared silicalite nanocrystals via hydrothermal synthesis using surfactants. Moreover, in our method, silicalite nanocrystals with a diameter of approximately $80 \mathrm{~nm}$ could be obtained in even a high concentration region of Si source. In this study, we demonstrate a method for preparing monodispersed silicalite nanocrystals in a solution consisting of surfactants, organic solvents, and water. The effects of the ionicity of the hydrophilic group in surfactant molecules and of the concentration of the Si source on the crystallinity and morphology of the nanocrystals were investigated.

Two kinds of solution were prepared in advance. Solution A was a water solution containing Si source. This was obtained by hydrolyzing metal alkoxide (tetraethylorthosilicate, TEOS) with a dilute tetrapropylammonium hydroxide (TPA-OH)/water solution at room temperature. The $\mathrm{pH}$ of the solution before crys- tallization was approximately 12.0. The concentration of TEOS and the molar ratio of TEOS to template were in the range of $0.5-2.7 \mathrm{~mol} / \mathrm{L}$ and 3.0-5.0, respectively. Solution B was an organic solution containing surfactant. A nonionic surfactant, polyoxyethylene (15) oleyl ether (O-15), and ionic surfactants, sodium bis(2-ethylhexyl) sulfosuccinate (AOT), and cetyltrimethylammonium bromide (CTAB), were employed in this study. Cyclohexane or 1-hexanol was used as an organic solvent. Solution $\mathrm{A}$ was added to solution $\mathrm{B}$ at $50^{\circ} \mathrm{C}$, and the mixture was magnetically stirred for $1 \mathrm{~h}$. The mixture was then placed in a Teflon-sealed stainless bottle, heated to $80-160^{\circ} \mathrm{C}$, and held at the desired temperature for $50 \mathrm{~h}$ with stirring to produce silicalite nanocrystals. The precipitate thus obtained was centrifuged, washed thoroughly with propanol, dried at $100^{\circ} \mathrm{C}$ overnight, and calcined under an air flow at $500{ }^{\circ} \mathrm{C}$ to remove the surfactant and the template. The morphology and crystallinity of the silicalite nanocrystals were investigated as functions of the types of surfactants and the concentration of Si source using a field-emission scanning electron microscope (FE-SEM; JEOL JSM6500F) and an X-ray diffractometer (XRD; JEOL JDX-8020). The pore structure of the nanocrystals was evaluated by a $\mathrm{N}_{2}$ adsorption and desorption method (BEL Japan Belsorp mini). Raman spectra of the nanocrystals were measured using the $532 \mathrm{~nm}$ line of an $\mathrm{Hg}$ laser.

The morphology of the silicalite nanocrystals prepared using various surfactants was investigated under the following conditions: a TOES concentration of 0.63 or $2.73 \mathrm{~mol} / \mathrm{L}$, hydrothermal temperature of 140 or $100^{\circ} \mathrm{C}$ and concentration of surfactant in organic solvent (solution B) of $0.50 \mathrm{~mol} / \mathrm{L}$. Figures 1 and 2 show FE-SEM photographs and X-ray diffraction patterns of
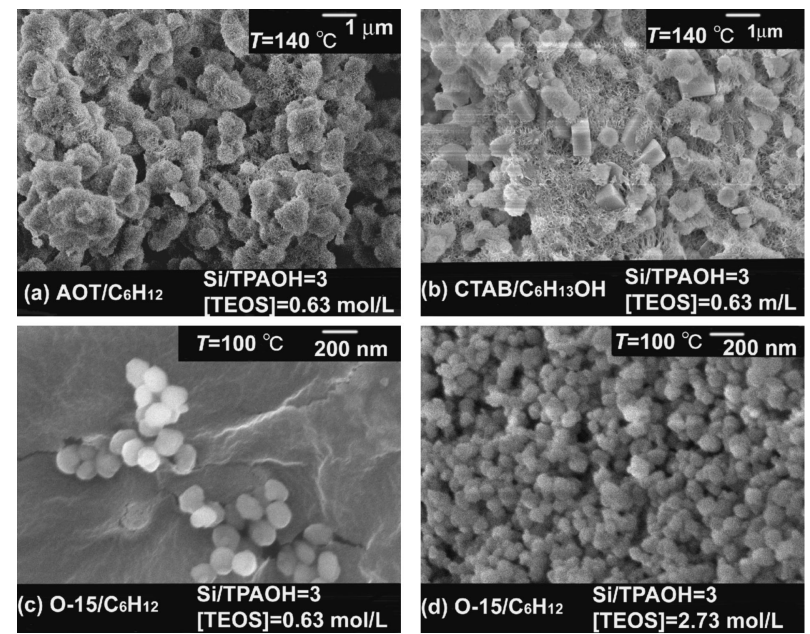

Figure 1. SEM photographs of samples prepared in various surfactant-organic solvent solutions. 


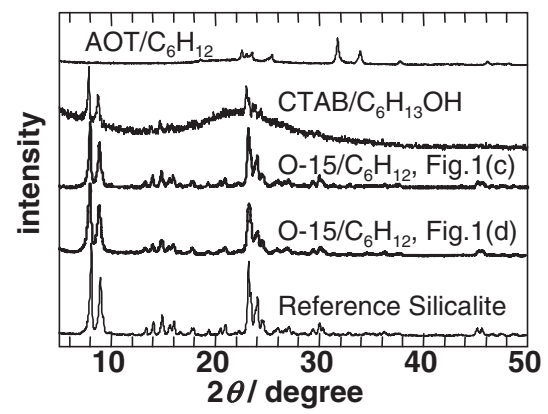

Figure 2. X-ray diffraction patterns of nanocrystals.

the obtained samples, respectively. The sample prepared in 1-hexanol solution containing AOT (AOT/cyclohexane) was irregular in shape. In the X-ray diffraction pattern of the sample prepared in AOT/cyclohexane solution, some peaks appeared, and these corresponded to sodium sulfate rather than silicalite. It was considered that the TPA-OH molecules could not act as a template in the synthetic solution. This result was ascribed to the fact that the surfactant of AOT and the template of TPA$\mathrm{OH}$ have opposite ionic charges. When using CTAB/1-hexanol, the silicalite crystals, which were approximately $1.0 \mu \mathrm{m}$ in size, were embedded in the amorphous $\mathrm{SiO}_{2}$ on the basis of the SEM observation. The coexistence of silicalite crystals and amorphous $\mathrm{SiO}_{2}$ was revealed by the X-ray diffraction analysis (Figure 2). Since the $\mathrm{pH}$ of the synthetic solution was alkaline, the surface of the $\mathrm{SiO}_{2}$ produced by hydrolysis of TEOS possessed a negative charge. Accordingly, it was considered that the CTAB and TPA-OH were independently adsorbed on the surface of $\mathrm{SiO}_{2}$ because of their cationic ionicity. Therefore, $\mathrm{SiO}_{2}$ species adsorbing TPA-OH and CTAB would change into silicalite crystals and amorphous $\mathrm{SiO}_{2}$, respectively. On the other hand, in the case of nonionic surfactants, O-15/cyclohexane, monodispersed silicalite nanocrystals were obtained, as shown in Figures 1c and 1d. The X-ray diffraction patterns of the samples showed peaks corresponding to pentasile-type zeolite (the reference silicalite in Figure 2). The average sizes of the silicalites were $120 \mathrm{~nm}$ and $80 \mathrm{~nm}$ at the TEOS concentrations of $0.63 \mathrm{~mol} / \mathrm{L}$ and $2.73 \mathrm{~mol} / \mathrm{L}$, respectively. On the contrary, SEM observation for silicalite crystal prepared in water (without surfactant) showed heterogeneous structure of smaller crystals (diameter of approximately $30 \mathrm{~nm}$ ) on a larger one (approximately $120 \mathrm{~nm}$ ), indicating that the nucleation, crystallization and crystal growth simultaneously occurred. These results indicated that the ionicity of the hydrophilic groups in the surfactant molecules played an important role in the formation and crystallization processes of the silicalite nanocrystals. Furthermore, the finding that the size of silicalites was dependent on the TEOS concentration was ascribed to the number of silicalite nuclei produced at the initial stage of hydrothermal synthesis. The increase in the TEOS concentration led to an increase in the number of silicalite nuclei, on which the nonionic surfactants were adsorbed. Accordingly, since the aggregation of the silicalite nuclei was inhibited by the adsorbed surfactants on the surface during hydrothermal treatment, monodispersed nanocrystals could be prepared.

Figure 3 shows the $\mathrm{N}_{2}$ adsorption isotherms of the silicalite nanocrystals, where $P$ is the adsorption equilibrium pressure and $P_{0}$ is the saturated vapor pressure of $\mathrm{N}_{2}$ at $77.3 \mathrm{~K}$. The steep in-

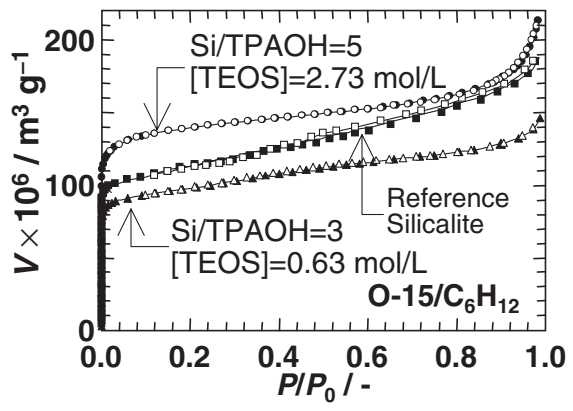

Figure 3. $\mathrm{N}_{2}$ adsorption and desorption isotherm of silicalite nanocrystals.

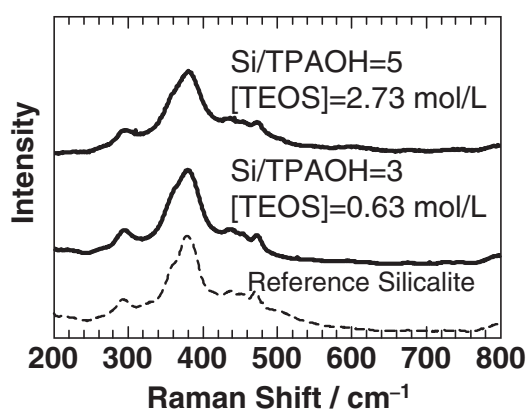

Figure 4. Raman spectra of silicalite nanocrystals.

crease in the amount of adsorbed $\mathrm{N}_{2}$ in the region of low relative pressure $\left(P / P_{0}\right)$, followed by the flat curve, corresponded to the filling of micropores. The adsorbed volumes of $\mathrm{N}_{2}$ for the nanocrystals were approximately $100 \times 10^{-6}-120 \times 10^{-6} \mathrm{~m}^{3} / \mathrm{g}$, indicating that the nanocrystals contained a considerable amount of micropore volume in their framework and were well crystallized.

Figure 4 shows the Raman spectrum of the nanocrystals. The peaks of the spectrum in the range of $300-650 \mathrm{~cm}^{-1}$ are indicative of the type of silicon-oxygen rings existing in the structure of zeolite. ${ }^{6,7}$ The spectrum of the nanocrystal showed peaks around 380,430 , and $470 \mathrm{~cm}^{-1}$, which corresponded to the five-, six-, and four-member rings, respectively. These peaks were in good agreement with the peaks of the reference silicalite prepared in water. In the structure of the MFI-type zeolite, continuous chains of five-member rings are connected by the fourand six-member rings. These results indicated that the surfaces of the nanocrystals with a diameter of approximately 120 and $80 \mathrm{~nm}$ were also well crystallized without amorphous $\mathrm{SiO}_{2}$.

\section{References}

1 S. Mintova, N. H. Olson, V. Valtchev, and T. Bein, Science, 283, 958 (1999).

2 R. Singh and P. K. Dutta, Langmuir, 16, 4148 (2000).

3 M. Tsapatsis, M. Lovallo, and M. E. Davis, Microporous Mater., 5, 381 (1996).

4 B. A. Holmberg, H. Wang, J. M. Norbeck, and Y. Yan, Microporous Mesoporous Mater., 59, 13 (2003).

5 H. Hosokawa and K. Oki, Chem. Lett., 32, 586 (2003).

6 Q. Li, B. Mihailova, D. Creaser, and J. Sterte, Microporous Mesoporous Mater., 43, 51 (2001).

7 P. K. Dutta, K. M. Rao, and J. Y. Park, J. Phys. Chem., 95, 6554 (1991). 